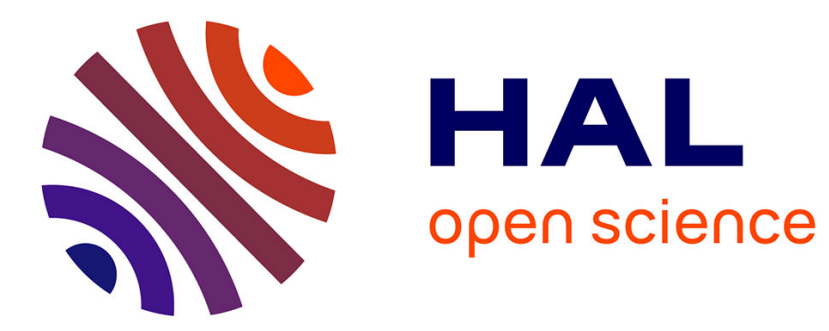

\title{
Functional Limit Theorem for the Empirical Process of a Class of Bernoulli Shifts with Long Memory
}

Paul Doukhan, Gabriel Lang, Donatas Surgailis, Marie Claude Viano

\section{To cite this version:}

Paul Doukhan, Gabriel Lang, Donatas Surgailis, Marie Claude Viano. Functional Limit Theorem for the Empirical Process of a Class of Bernoulli Shifts with Long Memory. Journal of Theoretical Probability, 2005, 18 (1), pp.161-186. 10.1007/s10959-004-2593-3 . hal-00267623

\section{HAL Id: hal-00267623 https://hal.science/hal-00267623}

Submitted on 27 Mar 2008

HAL is a multi-disciplinary open access archive for the deposit and dissemination of scientific research documents, whether they are published or not. The documents may come from teaching and research institutions in France or abroad, or from public or private research centers.
L'archive ouverte pluridisciplinaire HAL, est destinée au dépôt et à la diffusion de documents scientifiques de niveau recherche, publiés ou non, émanant des établissements d'enseignement et de recherche français ou étrangers, des laboratoires publics ou privés. 
Functional LIMIT THEOREM FOR THE EMPIRICAL PROCESS OF A CLASS OF BERNOULli SHIFTS WITH LONG MEMORY

Paul Doukhan ${ }^{1}$, Gabriel Lang ${ }^{2}$, Donatas Surgailis ${ }^{3}$ and Marie-Claude Viano ${ }^{4}$

${ }^{1}$ LS-CREST and University Cergy Pontoise, 95011 Cergy-Pontoise Cedex, France

${ }^{2}$ Laboratoire GRESE, ENGREF, 19 av. du Maine, 75732 Paris Cedex 15, France

${ }^{3}$ Vilnius Institute of Mathematics and Informatics, Akademijos 4, 2600 Vilnius, Lithuania

${ }^{4}$ Université de Lille 1, Laboratoire de Mathématiques Appliquées, Bt. M2,

Villeneuve d'Ascq, 59655 Cedex, France

\begin{abstract}
We prove a functional central limit theorem for the empirical process of a stationary process $X_{t}=Y_{t}+V_{t}$, where $Y_{t}$ is a long memory moving average in i.i.d. r.v.'s $\zeta_{s}, s \leq t$, and $V_{t}=V\left(\zeta_{t}, \zeta_{t-1}, \ldots\right)$ is a weakly dependent nonlinear Bernoulli shift. Conditions of weak dependence of $V_{t}$ are written in terms of $L^{2}$-norms of shift-cut differences $V\left(\zeta_{t}, \ldots, \zeta_{t-n}, 0, \ldots,\right)$ $V\left(\zeta_{t}, \ldots, \zeta_{t-n+1}, 0, \ldots\right)$. Examples of Bernoulli shifts are discussed. The limit empirical process is a degenerated process of the form $f(x) Z$, where $f$ is the marginal p.d.f. of $X_{0}$ and $Z$ is a standard normal r.v. The proof is based on a uniform reduction principle for the empirical process.
\end{abstract}

\title{
1 Introduction
}

Time series analysis has important statistical applications in various fields. For example, nonlinear times series are used to model crashes in financial markets.

The main object of times series analysis is the study of short-range dependent random sequences for which the usual Donsker and the Empirical Functional Limit Theorems (EFLT) hold with appropriate modifications. Rosenblatt (1961), in his seminal work, and afterwards, Taqqu (1975), Dobrushin and Major (1979) and other authors found that alternative 
limit behaviors may happen, in particular, non- $\sqrt{N}$ rates and non-central limits were exhibited. Most of these authors studied Gaussian subordinated case, or partial sums of nonlinear functions of a stationary Gaussian process with long-range dependence. The EFLT (with normalization $\ll N^{1 / 2}$ ) for such Gaussian subordinated processes was proved in Dehling and Taqqu (1989). An important feature of their EFLT is the fact that the limiting empirical process is degenerated, i.e. it has the form $g(x) Z$, with some deterministic function $g(x)$ and a random variable $Z$. Similar results for linear processes with long-range dependence were obtained in Giraitis, Koul and Surgailis (1996), Ho and Hsing (1996), Giraitis and Surgailis (1999) and other papers.

It is clear that Gaussian subordination or linearity are very restrictive structural assumptions which might be hard to justify in practice. In this paper we discuss the EFLT for a class of long-range dependent processes which are neither linear nor Gaussian subordinated. These are strictly stationary processes with discrete time $t \in \mathbb{Z}:=\{0, \pm 1, \pm 2, \ldots\}$ which can be represented as the sum

$$
X_{t}=Y_{t}+V_{t}
$$

of a linear long memory process $Y_{t}$ and (nonlinear) short memory process $V_{t}$ of a rather general form. More precisely, we assume that

$$
Y_{t}:=\sum_{i=0}^{\infty} b_{i} \zeta_{t-i}
$$

is a moving average process in i.i.d. random variables $\zeta_{i}, i \in \mathbb{Z}$ with zero mean and unit variance, with hyperbolically decaying coefficients

$$
b_{i} \sim c_{0} i^{d-1} \quad\left(\exists 0<d<1 / 2, c_{0} \neq 0\right) .
$$

The short memory process $V_{t}$ in (1.1) is the so-called Bernoulli shift:

$$
V_{t}:=V\left(\zeta_{t}, \zeta_{t-1}, \ldots\right)
$$

where $V\left(z_{0}, z_{1}, \ldots\right)$ is a Borel function on $\mathbb{R}^{\mathbb{Z}_{+}}, \mathbb{Z}_{+}=\{0,1, \ldots\}$. The short memory property of $V_{t}$ roughly means that the dependence of the function $V\left(z_{0}, z_{1}, \ldots\right)$ on coordinates $z_{n}$ is negligible with $n \rightarrow \infty$. Rigorous definition of the short memory property of $V_{t}$ involves $L^{2}$-norms of the shift-cut differences $V\left(\zeta_{0}, \ldots, \zeta_{-n}, 0, \ldots\right)-V\left(\zeta_{0}, \ldots, \zeta_{-n+1}, 0, \ldots\right)$, see Section 2, which must decrease sufficiently fast (e.g. subexponentially or hyperbolically) with $n$. 
Section 2 contains the main result of the paper (the EFLT for the empirical process of $X_{t}$ in (1.1)-(1.4)). Concrete examples of Bernoulli shifts $V_{t}$ in (1.4) are presented in Section 3. The remaining Sections 4-7 are given to the proof of the EFLT. It uses martingale techniques introduced in Ho and Hsing (1996) which were later applied by several authors.

Acknowledgement. The authors are grateful to the anonymous referee for careful reading and many useful comments.

\section{Main result}

Let $X_{1}, \ldots, X_{N}$ be the observed sample from the stationary process $X_{t}$ of (1.1). The empirical c.d.f. (empirical process)

$$
\hat{F}_{N}(x):=N^{-1} \sum_{t=1}^{N} I\left(X_{t} \leq x\right), \quad x \in \mathbb{R},
$$

is a consistent estimator of the marginal c.d.f. $F(x)=P\left[X_{0} \leq x\right]$. In fact, from ergodicity of the Bernoulli shift and the Glivenko-Cantelli theorem it follows that $\hat{F}_{N}(x) \rightarrow F(x)(N \rightarrow$ $\infty)$ uniformly in $x \in \mathbb{R}$ a.s. Write $f(x):=F^{\prime}(x)$ for the marginal p.d.f. of $X_{t}$, provided it exists. In addition to (1.3), we shall assume the following conditions on the innovations:

$$
\left|E e^{i u \zeta_{0}}\right| \leq C\left(1+u^{2}\right)^{-\delta} \quad(\exists C, 0<\delta \leq 1 / 4, \forall u \in \mathbb{R})
$$

and

$$
E\left|\zeta_{0}\right|^{3}<\infty
$$

We do not consider the "best" (i.e. the larger) $\delta$ available here. If the inequality in $(2.2)$ is satisfied for $\delta>1 / 4$ it is also satisfied for $\delta=1 / 4$. Note that condition (2.2) is very general because it does not imply that the density of $\zeta$ exists. But it excludes discrete distributions. If the inequality in (2.2) is satisfied with $\delta>1 / 4$, then the density exists in $L^{2}$, and if $\delta>1 / 2$ the density is bounded.

Put $\bar{Y}_{N}:=N^{-1} \sum_{t=1}^{N} Y_{t}$. Write $\Longrightarrow_{D(\overline{\mathbb{R}})}$ for weak convergence of random processes in the Skorohod space $D(\overline{\mathbb{R}}), \overline{\mathbb{R}}:=[-\infty, \infty]$ with the sup-norm topology, and $\Longrightarrow$ for weak convergence of finite dimensional distributions. Let

$$
\gamma_{n}:=E^{1 / 2}\left(V_{0}^{n}-V_{0}^{n-1}\right)^{2}
$$


where

$$
V_{t}^{n}:=V\left(\zeta_{t}, \ldots, \zeta_{t-n}, 0,0, \ldots\right)
$$

is the truncated Bernoulli shift (1.4) which is $(n+1)$-dependent stationary process.

Theorem 1 Assume conditions (1.2), (1.3), (2.2), (2.3). Moreover, let

$$
\gamma_{n} \leq C n^{-\rho}
$$

where

$$
\rho>\max \left\{24-22 d, 13-11 d+\frac{3(1-2 d)}{4 d}\right\} .
$$

Then

$$
\sup _{x \in \mathbb{R}} N^{(1 / 2)-d}\left|\hat{F}_{N}(x)-F(x)+f(x) \bar{Y}_{N}\right|=o_{p}(1) .
$$

Note that the maximum in the condition (2.7) is $24-22 d>13$ on most of the interval $d \in(0,1 / 2)$, with a change occurring very close to 0 at $d \approx 0.06$.

Theorem 1 is the uniform reduction principle for the empirical process (2.1) which extends the reduction principle of Dehling and Taqqu (1989) to Bernoulli shifts of the form (1.1)(1.4). It is well-known (Davydov (1970)) that the sample mean $\bar{Y}_{N}$ in (2.8) is asymptotically normal: $N^{(1 / 2)-d} \bar{Y}_{N} \Longrightarrow \tilde{c} Z$, where $Z \sim N(0,1)$ and $\tilde{c}:=\left(c_{0}^{2} B(d, 2-2 d) / d(1+2 d)\right)^{1 / 2}$, where $B(\cdot, \cdot)$ is the beta function. From this and the above theorem, it easily follows the EFLT below.

Corollary 2 Under conditions of Theorem 1,

$$
N^{(1 / 2)-d}\left(\hat{F}_{N}(x)-F(x)\right) \Longrightarrow_{D(\overline{\mathbb{R}})} \tilde{c} f(x) Z,
$$

where $Z \sim N(0,1)$.

The fact that the functional dependence of the limiting empirical process in (2.9) reduces to marginal p.d.f. of observable time series $X_{t}$ and does not involve probability densities or any other characteristics of unobservable components $Y_{t}$ and $V_{t}$, is rather surprising. It appears that many results in statistical inference of long memory processes which rely on the empirical process can be extended from linear or Gaussian models to the much more general class (1.1)-(1.4), and that "short memory perturbation" $V_{t}$ has no effect on large 
sample behavior of inferential procedures. It also seems that Theorem 1 can be extented to the asymptotic expansion of the empirical process similar to that given by Ho and Hsing (1996) in the case of linear process. This problem is closely related to the study of partial sums processes of nonlinear functions of $X_{t}$ in (1.1) and the characterization of their limiting behavior. In the case of Gaussian innovations $\zeta_{t}$ and finitely dependent $V_{t}$, this problem was studied in Surgailis (2000). Further possibilities involve nonadditive generalizations of (1.1) of the form $X_{t}=H\left(Y_{t} ; \zeta_{t}, \zeta_{t-1}, \ldots\right)$ and will be studied in another paper.

\section{Examples of weakly dependent Bernoulli shifts}

1. Volterra processes. A Volterra process is a stationary process defined through a convergent Volterra expansion $V_{t}=\sum_{k=1}^{\infty} V_{k ; t}$, where

$$
V_{k ; t}:=\sum_{0 \leq i_{1}<\ldots<i_{k}} a_{k ; i_{1}, \ldots, i_{k}} \zeta_{t-i_{1}} \ldots \zeta_{t-i_{k}}
$$

converges in $L^{2}$ provided the weights are square summable: $\sum_{0 \leq i_{1}<\ldots<i_{k}} a_{k ; i_{1}, \ldots, i_{k}}^{2}<\infty$. It is easy to see that in this case $\gamma_{n}=\left\{\sum_{k=1}^{n} \sum_{0 \leq i_{1}<\ldots<i_{k-1}<n} a_{k ; i_{1}, \ldots, i_{k-1}, n}^{2}\right\}^{1 / 2}$.

2. $A R C H(\infty)$ processes. A particular case of (non-Markovian) Bernoulli shifts is the $\operatorname{ARCH}(\infty)$ process (see Robinson (1991), Giraitis, Kokoszka and Leipus (2000), Giraitis and Surgailis (2002)). It is subject to the recursion equation

$$
V_{t}=\left(a_{0}+\sum_{j=1}^{\infty} a_{j} V_{t-j}\right) \zeta_{t}^{2}
$$

where $\zeta_{t}, t \in \mathbb{Z}$ are zero mean i.i.d. r.v.'s, as in (1.2), and $a_{j} \geq 0, j=0,1, \ldots$ are nonnegative coefficients. Put $\mu_{i}:=E \zeta_{0}^{2 i}, i \geq 1$. Under the simple condition

$$
\mu_{2}^{1 / 2} \sum_{i=1}^{\infty} a_{i}<1,
$$

equation (3.1) is known to have a unique stationary solution with finite variance and given by a convergent (although nonorthogonal) Volterra expansion

$$
V_{t}=a_{0} \zeta_{t}^{2}\left(1+\sum_{\ell=1}^{\infty} \sum_{s_{l}<\ldots<s_{1}<t} a_{t-s_{1}} \cdots a_{s_{\ell-1}-s_{\ell}} \zeta_{s_{1}}^{2} \cdots \zeta_{s_{\ell}}^{2}\right) .
$$

Below we assume (3.2) satisfied. Put

$$
g_{n}:=\sum_{\ell=1}^{n} \sum_{j_{1}+\ldots+j_{\ell}=n} \alpha_{j_{1}} \ldots \alpha_{j_{\ell}}
$$


where $\alpha_{n}:=\mu_{2}^{1 / 2} a_{n}$ and where the second sum is taken over all integers $j_{1}, \ldots, j_{\ell} \geq 1$ such that $j_{1}+\ldots+j_{\ell}=n$.

Proposition 1 For the ARCH( $\infty)$ process of (3.3),

$$
\gamma_{n}=E^{1 / 2}\left(V_{0}^{n}-V_{0}^{n-1}\right)^{2} \leq\left|a_{0}\right| \mu_{2} g_{n}
$$

Proof. We shall assume $a_{0}=1$ for simplicity. From (3.3) we obtain

$$
V_{0}^{n}=\zeta_{0}^{2}\left(1+\sum_{\ell=1}^{n} \sum_{-n \leq s_{\ell}<\ldots<s_{1}<0} a_{-s_{1}} \ldots a_{s_{\ell-1}-s_{\ell}} \zeta_{s_{1}}^{2} \ldots \zeta_{s_{\ell}}^{2}\right)
$$

and therefore

$$
V_{0}^{n}-V_{0}^{n-1}=\zeta_{0}^{2} \zeta_{-n}^{2} \sum_{\ell=1}^{n} \sum_{-n<s_{\ell-1}<\ldots<s_{1}<0} a_{-s_{1}} \ldots a_{s_{\ell-1}+n} \zeta_{s_{1}}^{2} \ldots \zeta_{s_{\ell-1}}^{2} .
$$

Therefore by Minkowski inequality,

$$
\begin{aligned}
\gamma_{n} & =\mu_{2} E^{1 / 2}\left\{\sum_{\ell=1}^{n} \sum_{-n<s_{\ell-1}<\ldots<s_{1}<0} a_{-s_{1}} \ldots a_{s_{\ell-1}+n} \zeta_{s_{1}}^{2} \ldots \zeta_{s_{\ell-1}}^{2}\right\}^{2} \\
& \leq \mu_{2} \sum_{\ell=1}^{n} \sum_{-n<s_{\ell-1}<\ldots<s_{1}<0} a_{-s_{1}} \ldots a_{s_{\ell-1}+n} E^{1 / 2} \zeta_{s_{1}}^{4} \ldots E^{1 / 2} \zeta_{s_{\ell-1}}^{4} \\
& =\mu_{2} \sum_{\ell=1}^{n} \sum_{0<s_{1}<\ldots<s_{\ell-1}<n} \alpha_{s_{1}} \ldots \alpha_{n-s_{\ell-1}}=\mu_{2} g_{n} .
\end{aligned}
$$

Inequality (3.5) allows to compare decay rates of $\gamma_{n}$ to those of $a_{n}$, or $\alpha_{n}$. Note the following relation between the generating series $G(z):=\sum_{n=1}^{\infty} g_{n} z^{n}, A(z):=\sum_{n=1}^{\infty} \alpha_{n} z^{n}$ :

$$
G(z)=\frac{A(z)}{1-A(z)}
$$

\section{Proposition 2}

(i) Let $a_{n}=O\left(p_{0}^{n}\right)$ for some $0<p_{0}<1$. Then there exists $0<p<1$ such that $\gamma_{n}=O\left(p^{n}\right)$.

(ii) Let $a_{n}=O\left(n^{-\lambda}\right)$ with some $\lambda>1$. Then $\gamma_{n}=O\left(n^{-\lambda}\right)$.

Proof. (i) Note $a_{n}=O\left(p_{0}^{n}\right)$ implies that $A(z)$ is analytic on $\left\{z \in \mathbb{C}:|z|<p_{0}^{-1}\right\}$. Moreover, $|A(z)| \leq \sum_{n=1}^{\infty} \alpha_{n}|z|^{n} \leq \sum_{n=1}^{\infty} \alpha_{n}<1$ for $|z| \leq 1$ and therefore $1-A(z) \neq 0$ for $|z|<c$ and some $c>1$. Therefore $G(z)$ is analytic on a disc of the complex plane of radius $>1$, which implies $\gamma_{n}=O\left(g_{n}\right)=O\left(p^{n}\right)$ for some $p<1$. 
(ii) Let $g_{n, \ell}:=\sum_{j_{1}+\ldots+j_{\ell}=n} \alpha_{j_{1}} \ldots \alpha_{j_{\ell}}$ so that $g_{n}=\sum_{\ell=1}^{n} g_{n, \ell}$. Let $0 \leq \alpha_{j} \leq K j^{-\lambda}(j \geq$ 1), $\sum_{j=1}^{\infty} \alpha_{j}=: \bar{\alpha}$. We shall prove that these assumptions imply

$$
g_{n, \ell} \leq D(\lambda) \ell^{3+\lambda} \bar{\alpha}^{\ell} n^{-\lambda}, \quad 1 \leq \ell \leq n, \quad n \geq 1,
$$

with some $D(\lambda) \geq K$ independent of $n, \ell$.

As $\bar{\alpha}<1$, so (3.7) implies (ii). Note that it suffices to prove (3.7) for $\bar{\alpha}=\sum_{j=1}^{\infty} \alpha_{j}=1$, in which case $\sum_{n=1}^{\infty} g_{n, \ell}=1$ for any $\ell \geq 1$.

We shall prove (3.7) by induction on $\ell \geq 1$. Note that for any $\ell_{0} \geq 1$ there exists $\tilde{D}\left(\ell_{0}\right)$ such that

$$
g_{n, \ell} \leq \tilde{D}\left(\ell_{0}\right) n^{-\lambda}, \quad 1 \leq \ell \leq \ell_{0}, \quad n \geq 1 .
$$

Relation (3.8) can be verified directly by definition of $g_{n, \ell}$ and the fact that $\lambda>1$. In view of (3.8), it suffices to show (3.7) for sufficiently large $\ell \geq \ell_{0}=\ell_{0}(\lambda)$ only.

From the the recurrent equation

$$
g_{n, \ell}=\sum_{j=1}^{n-1} \alpha_{n-j} g_{j, \ell-1}
$$

we obtain

$$
g_{n, \ell}=\sum_{n-(2 n /(2 \ell-1)) \leq j<n} \alpha_{n-j} g_{j, \ell-1}+\sum_{2 n /(2 \ell-1)<j<n} \alpha_{j} g_{n-j, \ell-1}=: g_{n, \ell}^{\prime}+g_{n, \ell}^{\prime \prime} .
$$

Here,

$$
g_{n, \ell}^{\prime \prime} \leq K(2 n /(2 \ell-1))^{-\lambda} \sum_{j=1}^{\infty} g_{j, \ell-1}=K(2 n /(2 \ell-1))^{-\lambda}=K(2 \ell-1)^{\lambda} 2^{-\lambda} n^{-\lambda} .
$$

On the other hand, by the inductive assumption,

$$
\begin{aligned}
g_{n, \ell}^{\prime} & \leq D(\lambda)(\ell-1)^{3+\lambda}(n(2 \ell-3) /(2 \ell-1))^{-\lambda} \sum_{j=1}^{\infty} \alpha_{j} \\
& =D(\lambda)(\ell-1)^{3+\lambda}(2 \ell-1)^{\lambda}(2 \ell-3)^{-\lambda} n^{-\lambda} .
\end{aligned}
$$

Therefore it suffices to check that for any $\ell>\ell_{0}$ and some $\ell_{0}=\ell_{0}(\lambda)>0$ large enough,

$$
D(\lambda)(\ell-1)^{3+\lambda}(2 \ell-1)^{\lambda}(2 \ell-3)^{-\lambda}+K(2 \ell-1)^{\lambda} 2^{-\lambda} \leq D(\lambda) \ell^{\lambda+3} .
$$

As $D(\lambda) \geq K$, the above inequality reduces to

$$
\left(1-\frac{1}{\ell}\right)^{3+\lambda}\left(1+\frac{2}{2 \ell-3}\right)^{\lambda}+\ell^{-3}\left(1-\frac{1}{2 \ell}\right)^{\lambda} \leq 1 .
$$


By taking Taylor expansion in $1 / \ell \rightarrow 0$, this gives

$$
-\frac{\lambda+3}{\ell}+\frac{2 \lambda}{2 \ell-3}+O\left(\ell^{-2}\right) \leq 0
$$

which is certainly true for $\ell>\ell_{0}(\lambda)$ large enough. This proves (3.7) and the proposition.

3. Stable Markov chains. It is well-known that a large class of Markov chains $V_{t}$ may be represented as a solution of recurrence equation

$$
V_{t}=M\left(V_{t-1}, \zeta_{t}\right)
$$

where $M(u, z)$ is a (measurable) kernel and $\left\{\zeta_{t}\right\}$ is an i.i.d. sequence (Kallenberg (1997)). Consider a more general situation when $V_{t}$ and $\zeta_{t}$ take values in Euclidean spaces $\mathbb{R}^{d}$ and $\mathbb{R}^{D}$, respectively, $d, D \geq 1$. Following Duflo (1990), we call (3.9) a Lipschitz Markov model if the kernel $M(u, z)$ satisfies

$$
E\left\|M\left(u, \zeta_{0}\right)-M\left(v, \zeta_{0}\right)\right\|^{2} \leq a\|u-v\|^{2}
$$

for all $u, v \in \mathbb{R}^{d}$ and some $a<1$, where $\|\cdot\|$ is a norm on $\mathbb{R}^{d}$. If in addition $M(u, 0)$ admits a fixed point $u_{0}$, one can show that a stationary and ergodic solution to (3.9) exists, which can written as a Bernoulli shift $V_{t}=V\left(\zeta_{t}, \zeta_{t-1}, \ldots\right)$; moreover, in this case (3.9), (3.10) imply

$$
\gamma_{n} \leq a^{n / 2} \gamma_{0}=a^{n / 2} E^{1 / 2}\left\|M\left(u_{0}, \zeta_{0}\right)-u_{0}\right\|^{2}
$$

so that $\gamma_{n}$ decay exponentially, due to $a<1$. Particular cases of (3.9) are ARCH-type processes (corresponding to kernels of the form $M(u, z)=A(u)+B(u) z)$, nonlinear $\operatorname{AR}(p)$ models, and many other processes. See Diaconis and Friedmann (1999), Doukhan (1994, 2002) for further examples and/or details.

\section{Plan of the proof of Theorem 1}

We first note that this theorem is known in the linear case $X_{t}=Y_{t}$, for $V_{t}=0$; see e.g. Ho and Hsing (1996) and Giraitis and Surgailis (1999). However, the proofs in our paper are essentially self-contained. Put $X_{t}^{n}:=Y_{t}+V_{t}^{n}$,

$$
\hat{F}_{N}^{n}(x):=N^{-1} \sum_{t=1}^{N} I\left(X_{t}^{n} \leq x\right), \quad F^{n}(x):=E \hat{F}_{N}^{n}(x)=P\left[X_{0}^{n} \leq x\right] .
$$


Let

$$
n(N):=N^{\lambda}, \quad \lambda \in(0,1),
$$

where $\lambda \in(0,1)$ will be specified below. The uniform reduction principle of (2.8) clearly follows from Lemmas 1 and 2 below. Everywhere below we suppose that the conditions of Theorem 1 are satisfied.

Lemma 1 If $\lambda<2 d$, then

$$
\sup _{x \in \mathbb{R}} N^{(1 / 2)-d}\left|\hat{F}_{N}^{n(N)}(x)-F^{n(N)}(x)+f(x) \bar{Y}_{N}\right|=o_{p}(1) .
$$

Lemma 2 If $\lambda>(3 / 2)(1-2 d) /(\rho+11 d-13)$, then

$$
\sup _{x \in \mathbb{R}} N^{(1 / 2)-d}\left(\left|\hat{F}_{N}^{n(N)}(x)-\hat{F}_{N}(x)\right|+\left|F^{n(N)}(x)-F(x)\right|\right)=o_{p}(1) .
$$

Remark 1 Note that condition (2.7) of Theorem 1 ensures that there exists $\lambda$ satisfying conditions of Lemmas 1 and 2 .

The proofs of the above lemmas require some bounds of marginal densities and their derivatives of the stationary processes $Y_{t}$ and $X_{t}$ and their approximations. These bounds are discussed in Section 5. Note that the short memory process $V_{t}$ need not have a density and its marginal distribution can be discrete. On the other hand, the long memory component $Y_{t}$ is known to have a smooth density under the hypotheses of Theorem 1 . As $V_{t}$ and $Y_{t}$ are dependent, the fact that the marginal density $f(x)$ of the sum $X_{t}=V_{t}+Y_{t}$ exists, is not trivial.

Notation. Put, for any $0 \leq n<m<\infty$,

$$
\begin{array}{rlrl}
Y_{t}^{n, m} & :=\sum_{i=n}^{m} b_{i} \zeta_{t-i}, & & G^{n, m}(x):=P\left[Y_{0}^{n, m} \leq x\right], \\
Y_{t}^{n, \infty}: & :=\sum_{i=n}^{\infty} b_{i} \zeta_{t-i}, \quad & G^{n, \infty}(x):=P\left[Y_{0}^{n, \infty} \leq x\right] .
\end{array}
$$

Let $G(x):=P\left[Y_{0} \leq x\right]$. Note $Y_{t}=Y_{t}^{0, n}+Y_{t}^{n+1, \infty}$, where $Y_{t}^{0, n}$ and $Y_{t}^{n+1, \infty}$ are independent for each $t, n$. Also, for $0<n \leq m<\infty$, put

$$
\begin{aligned}
X_{t}^{n} & :=Y_{t}+V_{t}^{n}, & F^{n}(x) & :=P\left[X_{0}^{n} \leq x\right], \\
X_{t}^{n, m} & :=Y_{t}^{0, m}+V_{t}^{n}, & F^{n, m}(x) & :=P\left[X_{0}^{n, m} \leq x\right] .
\end{aligned}
$$


We also write $g(x):=G^{\prime}(x), g^{n, m}(x):=\left(G^{n, m}(x)\right)^{\prime}, g^{n, \infty}(x):=\left(G^{n, \infty}(x)\right)^{\prime}, f^{n}(x):=$ $\left(F^{n}(x)\right)^{\prime}, f^{n, m}(x):=\left(F^{n, m}(x)\right)^{\prime}$ for the corresponding probability densities, provided they exist, and

$$
\begin{aligned}
& \hat{g}(u):=E e^{i u Y_{0}}, \quad \hat{g}^{n, m}(u):=E e^{i u Y_{0}^{n, m}}, \quad \hat{g}^{n, \infty}(u):=E e^{i u Y_{0}^{n, \infty},} \\
& \hat{f}(u):=E e^{i u X_{0}}, \quad \hat{f}^{n, m}(u):=E e^{i u X_{0}^{n, m}}, \quad \hat{f}^{n}(u):=E e^{i u X_{0}^{n}}
\end{aligned}
$$

for the characteristic functions. We also use the notation $\hat{\psi}(u)=\int_{\mathbb{R}} e^{i u x} \psi(x) d x, u \in \mathbb{R}$ for the Fourier transform of an integrable function $\psi=\psi(x), x \in \mathbb{R}$. Put

$$
\Phi(x):=P\left[\zeta_{0} \leq x\right], \quad \hat{\phi}(u):=E e^{i u \zeta_{0}} .
$$

In the sequel, $C$ stands for generic constant which may change from line to line.

\section{Bounds of marginal densities}

Put

$$
B_{n, j}:=\prod_{i=n}^{n+j} b_{i}^{2} .
$$

Note that, by (1.3), for each $j=0,1, \ldots$

$$
B_{n, j} \sim c_{0}^{2(1+j)} n^{2(d-1)(1+j)}, \quad n \rightarrow \infty .
$$

In particular, for any fixed $j \geq 0$ there exists a constant $C>0$ such that for all sufficiently large $n$,

$$
B_{n, j}^{-1} \leq C n^{2(1-d)(1+j)}
$$

Lemma 3 For any $p=0,1, \ldots$ there exist an integer $j_{0}=j_{0}(p) \geq 1$ and a constant $C=C(p)<\infty$ such that for all $0 \leq n<m, m-n \geq j_{0}$ and any $k=0,1,2$,

$$
\left|\left(u^{p} \hat{g}^{n, m}(u)\right)^{(k)}\right|+\left|\left(u^{p} \hat{g}^{n, \infty}(u)\right)^{(k)}\right| \leq C n^{\theta}\left(1+u^{2}\right)^{-3}
$$

where $(\cdot)^{(k)}$ denotes the $k$ th derivative. Moreover,

$$
\mid\left(u^{p}\left(\hat{g}^{n, m}(u)-\hat{g}^{n, m-1}(u)\right)^{(k)} \mid \leq C b_{m}^{2} n^{\theta}\left(1+u^{2}\right)^{-2}\right.
$$

where $\theta:=(1-d)(9+p)$. 
Proof. Let us prove (5.2) for $k=0$. Without loss of generality, assume $\left|b_{i}\right| \leq 1 \forall i$. By (2.2),

$$
\begin{aligned}
\left|\hat{g}^{n, m}(u)\right| \leq \prod_{i=n}^{n+j_{0}}\left|\hat{\phi}\left(u b_{i}\right)\right| & \leq C\left(\prod_{i=n}^{n+j_{0}}\left(1+u^{2} b_{i}^{2}\right)^{-1}\right)^{\delta} \\
& \leq C\left(\prod_{i=n}^{n+j_{0}} b_{i}^{-2}\left(1+u^{2}\right)^{-1}\right)^{\delta}=C B_{n, j_{0}}^{-\delta}\left(1+u^{2}\right)^{-\left(j_{0}+1\right) \delta} .
\end{aligned}
$$

Hence

$$
\left|u^{p} \hat{g}^{n, m}(u)\right| \leq C B_{n, j_{0}}^{-\delta}\left(1+u^{2}\right)^{p / 2-\left(j_{0}+1\right) \delta} .
$$

Taking $j_{0}=[(6+p) / 2 \delta]$ leads to $p / 2-\left(j_{0}+1\right) \delta \leq-3$ and, by $(5.1)$,

$$
B_{n, j_{0}}^{-\delta} \leq C n^{(1-d)(6+p+2 \delta)},
$$

thereby proving (5.2) for $k=0$.

In a similar way, let us prove (5.2) for $k=1$ and $k=2$. By (2.3), the characteristic function $\hat{\phi}(\cdot)$ is three times continuously differentiable, and we can write

$$
\begin{aligned}
\left(\hat{g}^{n, m}(u)\right)^{\prime}= & \sum_{j=n}^{m} i b_{j} \hat{\phi}^{\prime}\left(u b_{j}\right) \prod_{i=n, i \neq j}^{m} \hat{\phi}\left(u b_{i}\right), \\
\left(\hat{g}^{n, m}(u)\right)^{\prime \prime}= & -\sum_{j=n}^{m} b_{j}^{2} \hat{\phi}^{\prime \prime}\left(u b_{j}\right) \prod_{i=n, i \neq j}^{m} \hat{\phi}\left(u b_{i}\right) \\
& -\sum_{j_{1}, j_{2}=n, j_{1} \neq j_{2}}^{m} b_{j_{1}} b_{j_{2}} \hat{\phi}^{\prime}\left(u b_{j_{1}}\right) \hat{\phi}^{\prime}\left(u b_{j_{2}}\right) \prod_{i=n, i \neq j_{1}, j_{2}}^{m} \hat{\phi}\left(u b_{i}\right) .
\end{aligned}
$$

Hence, using $|\hat{\phi}(u)| \leq 1,\left|\hat{\phi}^{\prime}(u)\right| \leq|u|,\left|\hat{\phi}^{\prime \prime}(u)\right| \leq 1$, as well as (1.3) and (2.2) we obtain

$$
\left|\left(\hat{g}^{n, m}(u)\right)^{\prime}\right| \leq \sum_{j=n}^{\infty} b_{j}^{2}|u| \prod_{i=n, i \neq j}^{n+j_{0}}\left|\hat{\phi}\left(u b_{i}\right)\right| \leq C B_{n, j_{0}-1}^{-\delta}|u|\left(1+u^{2}\right)^{-j_{0} \delta} .
$$

By taking $j_{0}=[(7+p) / 2 \delta]+1$ and noting that $B_{n, j_{0}-1}^{-\delta} \leq C n^{(1-d)(7+p+2 \delta)}$, we obtain

$$
\left|u^{p}\left(\hat{g}^{n, m}(u)\right)^{\prime}\right| \leq C B_{n, j_{0}-1}^{-\delta}\left(1+u^{2}\right)^{(p+1) / 2-j_{0} \delta} \leq C n^{\theta}\left(1+u^{2}\right)^{-3} .
$$

The last inequality together with $\left|\left(u^{p} \hat{g}^{n, m}(u)\right)^{\prime}\right| \leq C\left|u^{p-1} \hat{g}^{n, m}(u)\right|+\left|u^{p}\left(\hat{g}^{n, m}(u)\right)^{\prime}\right|$ and relation (5.2) for $k=0$ prove (5.2) for $k=1$. Similarly,

$$
\begin{aligned}
\left|\left(\hat{g}^{n, m}(u)\right)^{\prime \prime}\right| & \leq \sum_{j=n}^{\infty} b_{j}^{2} \prod_{i=n, i \neq j}^{n+j_{0}}\left|\hat{\phi}\left(u b_{i}\right)\right|+\sum_{j_{1}, j_{2}=n, j_{1} \neq j_{2}}^{\infty} u^{2} b_{j_{1}}^{2} b_{j_{2}}^{2} \prod_{i=n, i \neq j_{1}, j_{2}}^{n+j_{0}}\left|\hat{\phi}\left(u b_{i}\right)\right| \\
& \leq C\left(B_{n, j_{0}-1}^{-\delta}\left(1+u^{2}\right)^{-j_{0} \delta}+B_{n, j_{0}-2}^{-\delta} u^{2}\left(1+u^{2}\right)^{-\left(j_{0}-1\right) \delta}\right) \\
& \leq C B_{n, j_{0}-1}^{-\delta}\left(1+u^{2}\right)^{1-\left(j_{0}-1\right) \delta}
\end{aligned}
$$


Taking $j_{0}=[(8+p) / 2 \delta]+2$ and noting that $B_{n, j_{0}-1}^{-\delta} \leq C n^{2(1-d) \delta j_{0}} \leq C n^{(1-d)(8+p+4 \delta)}$, we obtain (5.2) for $k=2$.

It remains to prove (5.3). Let $k=0$. Since

$$
u^{p}\left(\hat{g}^{n, m}(u)-\hat{g}^{n, m-1}(u)\right)=\left(u^{p} \hat{g}^{n, m-1}(u)\right)\left(\hat{\phi}\left(b_{m} u\right)-1\right),
$$

inequality (5.3) follows from (5.2) and the bound $\left|\hat{\phi}\left(b_{m} u\right)-1\right| \leq b_{m}^{2} u^{2}$. Cases $k=$ 1,2 follow similarly. For instance, among the three terms appearing in the case $k=2$, $\left(u^{p} \hat{g}^{n, m-1}(u)\right)^{\prime \prime}\left(\hat{\phi}\left(b_{m} u\right)-1\right)$ leads to the value of $\theta$ given in the lemma. Indeed, using $(5.2)$,

$$
\left|\left(u^{p} \hat{g}^{n, m-1}(u)\right)^{\prime \prime}\left(\hat{\phi}\left(b_{m} u\right)-1\right)\right| \leq u^{2} b_{m}^{2}\left|\left(u^{p} \hat{g}^{n, m-1}(u)\right)^{\prime \prime}\right| \leq C b_{m}^{2} n^{\theta}\left(1+u^{2}\right)^{-2} .
$$

The lemma is proved.

Lemma 4 For any $p=0,1, \ldots$ there exist an integer $j_{0} \geq 1$ and a constant $C=C_{p}<\infty$ such that for all $0 \leq n<m, m-n \geq j_{0}, x \in \mathbb{R}$,

$$
\left|\left(g^{n, m}(x)\right)^{(p)}\right|+\left|\left(g^{n, \infty}(x)\right)^{(p)}\right| \leq C n^{\theta}\left(1+x^{2}\right)^{-1}
$$

and

$$
\left|\left(g^{n, m}(x)-g^{n, m-1}(x)\right)^{(p)}\right| \leq C b_{m}^{2} n^{\theta}\left(1+x^{2}\right)^{-1},
$$

where $\theta$ is the same as in the previous lemma.

Proof. Relation (5.4) follows from (5.2) and from

$$
\left(g^{n, m}(x)\right)^{(p)}=C \int e^{-i u x} u^{p} \hat{g}^{n, m}(u) d u, \quad x^{2}\left(g^{n, m}(x)\right)^{(p)}=C \int e^{-i u x}\left(u^{p} \hat{g}^{n, m}(u)\right)^{\prime \prime} d u .
$$

Similarly, (5.5) follows from (5.3).

Next we consider bounds for p.d.f. $f(x), f^{n}(x), f^{n, m}(x)$ of $X_{t}, X_{t}^{n}=V_{t}^{n}+Y_{t}, X_{t}^{n, m}=$ $V_{t}^{n}+Y_{t}^{0, m}$, respectively. To that end, we need elementary Lemma 5 below which is similar to Doukhan, Lang and Surgailis (2002, Lemmas 4.1, 4.2). Let

$$
\varphi_{r}(x):=(1+|x|)^{-r}, \quad \mu_{r}(x, y):=\int_{x}^{y} \varphi_{r}(z) d z, \quad x<y, r>1, x, y \in \mathbb{R} .
$$

Note $\mu_{r}$ is a finite measure on $\mathbb{R}$. 
Lemma 5 Let $h(x), x \in \mathbb{R}$ be a real valued function such that

$$
|h(x)| \leq C \varphi_{r}(x), \quad|h(x)-h(y)| \leq C|x-y| \varphi_{r}(x),
$$

hold for any $x, y \in \mathbb{R},|x-y| \leq 1$ and some $C<\infty, 1<r \leq 2$. Then there exists a constant $C_{r}$ depending only on $r$ and $C$ in (5.6), such that for any $x, y, v, z \in \mathbb{R}$

$$
\begin{aligned}
|h(x+y)| & \leq C \varphi_{r}(x)\left(1 \vee|y|^{r}\right), \\
\left|\int_{0}^{y} h(x+w) d w\right| & \leq C_{r} \varphi_{r}(x)\left(|y| \vee|y|^{r}\right), \\
\left|\int_{x}^{y}(h(\xi+v)-h(\xi)) d \xi\right| & \leq C_{r} \mu_{r}(x, y)\left(|v| \vee|v|^{r}\right), \\
\left|\int_{0}^{v} d w \int_{x}^{y}(h(\xi+w-z)-h(\xi-z)) d \xi\right| & \leq C_{r} \mu_{r}(x, y)|v|^{r}\left(1 \vee|z|^{r}\right) .
\end{aligned}
$$

Lemma 6 Let $p_{0}$ be a nonnegative integer and $\theta=(1-d)\left(9+p_{0}\right)$. If

$$
\sum_{n=1}^{\infty} n^{\theta} \gamma_{n}^{1 / 2}<\infty
$$

then for any $p \in\left\{0, \ldots, p_{0}\right\}$ and any $1<r<3 / 2$, there exist $j_{0} \geq 1$ and a constant $C=C_{p, r}<\infty$ such that for all $0 \leq n<m, m-n \geq j_{0}, x \in \mathbb{R}$

$$
\left|(f(x))^{(p)}\right|+\left|\left(f^{n}(x)\right)^{(p)}\right|+\left|\left(f^{n, m}(x)\right)^{(p)}\right| \leq C(1+|x|)^{-r}
$$

and, moreover,

$$
\left|(f(x))^{(p)}-\left(f^{n}(x)\right)^{(p)}\right| \leq C \alpha_{n}(1+|x|)^{-r}
$$

and

$$
\left|\left(f^{n}(x)\right)^{(p)}-\left(f^{n, m}(x)\right)^{(p)}\right| \leq C \beta_{m}^{1 / 2}(1+|x|)^{-r},
$$

where $\alpha_{n}:=\sum_{j=n}^{\infty} j^{\theta} \gamma_{j}^{1 / 2}, \beta_{n}:=\sum_{j=n}^{\infty} b_{j}^{2}$.

Proof. We shall prove (5.12) for $f^{n}(x)$ and $p=0$ only, as the remaining inequalities can be proved analogously. Let

$$
\psi^{n}(x):=f^{n}(x)-f^{n-1}(x),
$$

$n \geq 0, f^{-1}(x):=g(x)$. Clearly (5.12) (for $f^{n}(x)$ and $p=0$ ) follows from

$$
(1+|x|)^{r}\left|\psi^{n}(x)\right| \leq C n_{+}^{\theta} \gamma_{n}^{1 / 2},
$$


where $n_{+}:=n \vee 1, \gamma_{0}:=1$. To show (5.15), consider the Fourier transform

$$
\begin{aligned}
\left|\hat{\psi}^{n}(u)\right| & =\left|E e^{i u Y_{0}^{n+1, \infty}}\left(E e^{i u\left(Y_{0}^{0, n}+V_{0}^{n}\right)}-E e^{i u\left(Y_{0}^{0, n}+V_{0}^{n-1}\right)}\right)\right| \\
& =\left|\hat{g}^{n+1, \infty}(u)\right|\left|E e^{i u\left(Y_{0}^{0, n}+V_{0}^{n-1}\right)}\left(e^{i u\left(V_{0}^{n}-V_{0}^{n-1}\right)}-1\right)\right| \\
& \leq\left|\hat{g}^{n+1, \infty}(u)\right| E\left|e^{i u\left(V_{0}^{n}-V_{0}^{n-1}\right)}-1\right| \\
& \leq\left|\hat{g}^{n+1, \infty}(u)\right||u| E^{1 / 2}\left(V_{0}^{n}-V_{0}^{n-1}\right)^{2} \\
& \leq C n^{\theta} \gamma_{n}\left(1+u^{2}\right)^{-2}, \quad n \geq 1,
\end{aligned}
$$

where in the last line we used (5.2) with $p=1, k=0$. Also, $\left|\hat{\psi}^{0}(u)\right|=\left|\hat{f}^{0}(u)-\hat{f}^{-1}(u)\right|=$ $\left|E e^{i u Y_{0}^{1, \infty}}\right|\left|E e^{i u \zeta_{0}}\left(e^{i u V_{0}^{0}}-1\right)\right| \leq 2\left|\hat{g}^{1, \infty}(u)\right| \leq C\left(1+u^{2}\right)^{-3}$ according to (5.2). This proves $\left|\psi^{n}(x)\right| \leq C n_{+}^{\theta} \gamma_{n}, n \geq 0$. To show (5.15), it remains to prove that there exist constants $C, c>0$ such that

$$
|x|^{r}\left|\psi^{n}(x)\right| \leq C n_{+}^{\theta} \gamma_{n}^{1 / 2}, \quad|x| \geq c .
$$

The proof of (5.16) is more complicated as $r$ is not an integer; c.f. Doukhan, Lang and Surgailis (2002). To that end, we will show that there exists a (complex-valued) function $q(x)$ satisfying

$$
|q(x)| \geq c_{1}|x|^{r-1}, \quad|x| \geq c,
$$

where $c, c_{1}>0$ are some constants, and such that

$$
\left|q(x) x \psi^{n}(x)\right| \leq C n_{+}^{\theta} \gamma_{n}^{1 / 2}, \quad x \in \mathbb{R}, n \geq 0 .
$$

Similarly as in the above mentioned paper, take

$$
\begin{aligned}
q(x) & :=\int_{0}^{\infty}\left(1-e^{-i x z}\right) z^{-r} d z-\int_{1}^{\infty}\left(1-e^{-i x z}\right) z^{-r} d z \\
& =|x|^{r-1} e^{i \operatorname{sgn}(x) \pi(r-1) / 2}-\tilde{q}(x)
\end{aligned}
$$

where $\tilde{q}(x):=\int_{1}^{\infty}\left(1-e^{-i x z}\right) z^{-r} d z$ is a bounded function on the real line. Therefore $q(x)$ satisfies (5.17). To show (5.18), note that by Parseval's identity,

$$
q(x) \phi(x)=C \int_{\mathbb{R}} e^{-i u x} d u \int_{0}^{1}(\hat{\phi}(u)-\hat{\phi}(u-\xi)) \xi^{-r} d \xi
$$

for any smooth and integrable test function $\phi$. Consequently,

$$
q(x)(i x) \psi^{n}(x)=C \int_{\mathbb{R}} e^{-i u x} d u \int_{0}^{1}\left(\left(\hat{\psi}^{n}\right)^{\prime}(u)-\left(\hat{\psi}^{n}\right)^{\prime}(u-\xi)\right) \xi^{-r} d \xi .
$$


Here,

$$
\hat{\psi}^{n}(u)=\hat{g}^{n+1, \infty}(u) E\left(e^{i u X_{0}^{n-1, n}}\left(e^{i u\left(V_{0}^{n}-V_{0}^{n-1}\right)}-1\right)\right) \equiv h(u) a(u),
$$

where

$$
h(u):=\hat{g}^{n+1, \infty}(u), \quad a(u):=E e^{i u X}\left(e^{i u \Delta V}-1\right), \quad X:=X_{0}^{n-1, n}, \quad \Delta V:=V_{0}^{n}-V_{0}^{n-1} .
$$

Next,

$$
\begin{aligned}
\left(\hat{\psi}^{n}\right)^{\prime}(u)-\left(\hat{\psi}^{n}\right)^{\prime}(u-\xi) & =h^{\prime}(u) a(u)+h(u) a^{\prime}(u)-h^{\prime}(u-\xi) a(u-\xi)-h(u-\xi) a^{\prime}(u-\xi) \\
& =\left(h^{\prime}(u)-h^{\prime}(u-\xi)\right) a(u)+(h(u)-h(u-\xi)) a^{\prime}(u) \\
& +h^{\prime}(u-\xi)(a(u)-a(u-\xi))+h(u-\xi)\left(a^{\prime}(u)-a^{\prime}(u-\xi)\right) .
\end{aligned}
$$

From Lemma 3, (5.2) and Lemma 5, (5.7), it easily follows that

$$
\begin{aligned}
|h(u-\xi)| & \leq C n^{\theta}\left(1+u^{2}\right)^{-3}, \\
\left|h^{\prime}(u-\xi)\right| & \leq C n^{\theta}\left(1+u^{2}\right)^{-3}, \\
|h(u)-h(u-\xi)| & \leq C n^{\theta}|\xi|\left(1+u^{2}\right)^{-3}, \\
\left|h^{\prime}(u)-h^{\prime}(u-\xi)\right| & \leq C n^{\theta}|\xi|\left(1+u^{2}\right)^{-3},
\end{aligned}
$$

where the constant $C$ does not depend on $n, u \in \mathbb{R}, \xi \in(0,1)$. Next, consider $a(u), a^{\prime}(u), a(u)-$ $a(u-\xi), a^{\prime}(u)-a^{\prime}(u-\xi)$. We have

$$
|a(u)|=\left|E e^{i u X}\left(e^{i u \Delta V}-1\right)\right| \leq|u| E|\Delta V| \leq|u| \gamma_{n} .
$$

Similarly,

$\left|a^{\prime}(u)\right| \leq\left|E e^{i u X} X\left(e^{i u \Delta V}-1\right)\right|+\left|E e^{i u X} e^{i u \Delta V} \Delta V\right| \leq|u| E|X \Delta V|+E|\Delta V| \leq C(1+|u|) \gamma_{n}$,

where we used Cauchy-Schwarz inequality and the fact that

$$
E X^{2} \equiv E\left(X_{0}^{n-1, n}\right)^{2} \leq C
$$

Next,

$$
\begin{aligned}
|a(u)-a(u-\xi)| & =\left|E e^{i u X}\left\{\left(e^{i u \Delta V}-1\right)\left(1-e^{-i \xi(X+\Delta V)}\right)+e^{-i \xi X}\left(1-e^{-i \xi \Delta V}\right)\right\}\right| \\
& \leq|u \xi| E|\Delta V|(|X|+|\Delta V|)+|\xi| E|\Delta V| \leq C|\xi|(1+|u|) \gamma_{n},
\end{aligned}
$$


where we used $\gamma_{n}^{2} \leq C \gamma_{n}$. Finally, consider the most delicate term

$$
\begin{aligned}
\left|a^{\prime}(u)-a^{\prime}(u-\xi)\right| & =\mid E e^{i u X}(i X)\left\{\left(e^{i u \Delta V}-1\right)\left(1-e^{-i \xi(X+\Delta V)}\right)+e^{-i \xi X}\left(1-e^{-i \xi \Delta V}\right)\right\} \\
& -E e^{i u(X+\Delta V)}(i \Delta V)\left(e^{-i \xi(X+\Delta V)}-1\right) \mid \\
& \leq E|X|(2 \wedge|u \Delta V|)(2 \wedge|\xi(X+\Delta V)|)+|\xi| E|X||\Delta V| \\
& +|\xi| E|\Delta V|(|X|+|\Delta V|) .
\end{aligned}
$$

To evaluate the first expectation on the r.h.s., use the inequality $2 \wedge x \leq(2 x)^{1 / 2}$, then by Cauchy-Schwartz inequality,

$$
\begin{aligned}
E|X|(2 \wedge|u \Delta V|)(2 \wedge|\xi X|) & \leq 2|u \xi|^{1 / 2} E\left(|X|^{3 / 2}|\Delta V|^{1 / 2}\right) \\
& \leq 2|u \xi|^{1 / 2}\left(E|X|^{2}\right)^{3 / 4}\left(E|\Delta V|^{2}\right)^{1 / 4} \\
& \leq C|u \xi|^{1 / 2} \gamma_{n}^{1 / 2}
\end{aligned}
$$

Similarly, $E|X|(2 \wedge|u \Delta V|)(2 \wedge|\xi \Delta V|) \leq 2|u \xi|^{1 / 2} E|X||\Delta V| \leq C|u \xi|^{1 / 2} \gamma_{n}$ and we obtain

$$
\left|a^{\prime}(u)-a^{\prime}(u-\xi)\right| \leq C|\xi|^{1 / 2}(1+|u|) \gamma_{n}^{1 / 2}
$$

Combining the above bounds (recall that $|\xi|<1$ ) results in

$$
\left|\left(\hat{\psi}^{n}\right)^{\prime}(u)-\left(\hat{\psi}^{n}\right)^{\prime}(u-\xi)\right| \leq C|\xi|^{1 / 2} n^{\theta} \gamma_{n}^{1 / 2}\left(1+u^{2}\right)^{-2}
$$

yielding

$$
\left|q(x)(i x) \psi^{n}(x)\right| \leq C n^{\theta} \gamma_{n}^{1 / 2} \int_{\mathbb{R}}\left(1+u^{2}\right)^{-2} d u \int_{0}^{1} \xi^{1 / 2-r} d \xi \leq C n^{\theta} \gamma_{n}^{1 / 2},
$$

for any $1<r<3 / 2$. This proves (5.16) and (5.15), hence also (5.12) for $f^{n}(x)$ and $p=0$, with any $1<r<3 / 2$. Clearly, (5.15) implies (5.13) as well.

It remains to prove (5.14), where we again restrict ourselves to the case $p=0$, as the case $p \geq 1$ is analogous. We have

$$
f^{n}(x)-f^{n, m}(x)=\int_{\mathbb{R}}\left(f^{n, m}(x-y)-f^{n, m}(x)\right) g^{m+1, \infty}(y) d y .
$$

By (5.12),

$$
\begin{aligned}
& \int_{|y| \leq 1}\left|f^{n, m}(x-y)-f^{n, m}(x)\right| g^{m+1, \infty}(y) d y \leq C(1+|x|)^{-r} \int_{|y| \leq 1}|y| g^{m+1, \infty}(y) d y \\
& =C(1+|x|)^{-r} E\left|Y_{0}^{m+1, \infty}\right| \leq C(1+|x|)^{-r} E^{1 / 2}\left|Y_{0}^{m+1, \infty}\right|^{2}=C(1+|x|)^{-r} \beta_{m+1}^{1 / 2} .
\end{aligned}
$$


Similarly, by (5.12) and Lemma 5 (5.7),

$$
\begin{aligned}
& \int_{|y|>1} f^{n, m}(x-y) g^{m+1, \infty}(y) d y \leq C(1+|x|)^{-r} \int_{|y|>1}|y|^{r} g^{m+1, \infty}(y) d y \\
& \leq C(1+|x|)^{-r} E\left|Y_{0}^{m+1, \infty}\right|^{r} \leq C(1+|x|)^{-r}\left(E\left|Y_{0}^{m+1, \infty}\right|^{2}\right)^{r / 2} \\
& =C(1+|x|)^{-r} \beta_{m+1}^{r / 2} .
\end{aligned}
$$

A similar estimate holds for the integral $\int_{|y|>1} f^{n, m}(x) g^{m+1, \infty}(y) d y$. As $\beta_{m+1}^{r / 2} \leq C \beta_{m+1}^{1 / 2}$ for $r>1$, this proves the bound (5.14) for $p=0$.

Remark 2 In the sequel, we use Lemma 6 with $p_{0}=2$ and $\theta=11(1-d)$ only.

\section{Proof of Lemma 1}

Lemma 1 follows from Lemma 7 below combined with a standard chaining argument as in Dehling and Taqqu (1989) or Giraitis, Koul and Surgailis (1996, proof of Th. 1). For any function $\psi(x)$, and any $x<y$, put $\psi(x, y):=\psi(y)-\psi(x)$.

Lemma 7 Assume condition (2.6), where

$$
\rho>24-22 d \text {. }
$$

Let $n(N):=N^{\lambda}, 0<\lambda<2 d$. Define

$$
\kappa:=\min \{2 d-\lambda, 1-2 d, \lambda(\rho+22 d-24)\}
$$

There exists a finite measure $\mu=\mu_{r}(1<r<3 / 2)$ and a constant $C<\infty$ such that for all $N \geq 1, x<y$

$$
E\left|\hat{F}_{N}^{n(N)}(x, y)-F^{n(N)}(x, y)+f(x, y) \bar{Y}_{N}\right|^{2} \leq C \mu(x, y) N^{2 d-1-\kappa},
$$

Proof. Put

$$
S_{N}^{n}(x):=N\left(\hat{F}_{N}^{n}(x)-F^{n}(x)+f(x) \bar{Y}_{N}\right)=\sum_{t=1}^{N} R_{t}^{n}(x),
$$

where $R_{t}^{n}(x):=I\left(X_{t}^{n} \leq x\right)-F^{n}(x)+f(x) Y_{t}$. By the telescoping identity due to Ho and Hsing (1996), for any $m_{0}>0$,

$$
R_{t}^{n}(x)=\sum_{m \geq m_{0}} U_{t}^{n, m}(x),
$$


where

$$
\begin{aligned}
U_{t}^{n, m_{0}}(x) & :=I\left(X_{t}^{n} \leq x\right)-P\left[X_{t}^{n} \leq x \mid \mathcal{B}_{t-m_{0}-1}\right]+f(x) Y_{t}^{0, m_{0}}, \\
U_{t}^{n, m}(x) & :=P\left[X_{t}^{n} \leq x \mid \mathcal{B}_{t-m}\right]-P\left[X_{t}^{n} \leq x \mid \mathcal{B}_{t-m-1}\right]+f(x) b_{m} \zeta_{t-m}, \quad m>m_{0},
\end{aligned}
$$

where $\mathcal{B}_{s}:=\sigma\left\{\zeta_{u}, u \leq s\right\}$ is the history $\sigma$-field. Clearly, $E\left(S_{N}^{n}(x, y)\right)^{2} \leq 2\left(E\left(S_{N 0}^{n}(x, y)\right)^{2}+\right.$ $\left.E\left(S_{N 1}^{n}(x, y)\right)^{2}\right)$, where

$$
S_{N 0}^{n}(x, y):=\sum_{t=1}^{N} U_{t}^{n, m_{0}}(x, y), \quad S_{N 1}^{n}(x, y):=\sum_{t=1}^{N} \sum_{m>m_{0}} U_{t}^{n, m}(x, y) .
$$

We claim that there exists $1<r<3 / 2, j_{0} \geq 1$ and a constant $C<\infty$ such that for any $0 \leq n<m_{0}, m_{0}-n \geq j_{0}$, and any $x<y$

$$
\begin{aligned}
& E\left(S_{N 0}^{n}(x, y)\right)^{2} \leq C \mu_{r}(x, y) N m_{0}, \\
& E\left(S_{N 1}^{n}(x, y)\right)^{2} \leq C \mu_{r}(x, y)\left(\Theta(N)+N^{1+2 d} \alpha_{n}^{2}\right),
\end{aligned}
$$

where $\alpha_{n}$ is defined in Lemma 6 and

$$
\Theta(N):= \begin{cases}N, & \text { if } d<1 / 4 \\ N(\log N)^{2}, & \text { if } d=1 / 4 \\ N^{4 d}, & \text { if } d>1 / 4\end{cases}
$$

The claim (6.3), (6.4) is proved below. To conclude the statement of the lemma, note $\alpha_{n}^{2}=O\left(n^{24-22 d-\rho}\right)$ as $\theta=11(1-d)$, see Remark 2. Hence $\alpha_{n(N)}^{2}=O\left(N^{-\lambda(\rho+22 d-24)}\right)$ and $m_{0}(N)=O(n(N))=O\left(N^{\lambda}\right)$, implying $E\left(S_{N}^{n(N)}(x, y)\right)^{2} \leq C \mu_{r}(x, y) N^{1+2 d-\kappa}$, or $(6.2)$, with $\kappa$ given in Lemma 7 .

To prove (6.3) and (6.4), note by the orthogonality property of conditional expectations, for any $x, y$

$$
\begin{aligned}
E\left[U_{t}^{n, m_{0}}(x) U_{t^{\prime}}^{n, m_{0}}(y)\right] & =0, \quad\left|t-t^{\prime}\right|>m_{0}, \\
E\left[U_{t}^{n, m}(x) U_{t^{\prime}}^{n, m^{\prime}}(y)\right] & =0, \quad t-m \neq t^{\prime}-m^{\prime}, m, m^{\prime}>m_{0} .
\end{aligned}
$$

Then

$$
\begin{aligned}
E\left(S_{N 0}^{n}(x, y)\right)^{2} & =\sum_{1 \leq t, t^{\prime} \leq N,\left|t^{\prime}-t\right| \leq m_{0}} E U_{t}^{n, m_{0}}(x, y) U_{t^{\prime}}^{n, m_{0}}(x, y) \\
E\left(S_{N 1}^{n}(x, y)\right)^{2} & =\sum_{t, t^{\prime}=1}^{N} \sum_{m>m_{0}, t^{\prime}-t+m>m_{0}} E U_{t}^{n, m}(x, y) U_{t^{\prime}}^{n, t^{\prime}-t+m}(x, y) \\
& \leq \sum_{t, t^{\prime}=1}^{N} \sum_{m>m_{0}, t^{\prime}-t+m>m_{0}} E^{1 / 2}\left(U_{t}^{n, m}(x, y)\right)^{2} E^{1 / 2}\left(U_{t^{\prime}}^{n, t^{\prime}-t+m}(x, y)\right)^{2} .
\end{aligned}
$$


Clearly, by stationarity,

$$
\begin{aligned}
E\left(S_{N 0}^{n}(x, y)\right)^{2} & \leq \sum_{1 \leq t, t^{\prime} \leq N,\left|t^{\prime}-t\right| \leq m_{0}} E^{1 / 2}\left(U_{t}^{n, m_{0}}(x, y)\right)^{2} E^{1 / 2}\left(U_{t^{\prime}}^{n, m_{0}}(x, y)\right)^{2} \\
& \leq C N m_{0} E\left(U_{0}^{n, m_{0}}(x, y)\right)^{2}
\end{aligned}
$$

where the constant $C$ does not depend on $n, m_{0}, N$. Using definition of $U_{t}^{n, m_{0}}(x, y)$ together with Lemma 6 (5.12), we obtain

$$
\begin{aligned}
E\left(U_{0}^{n, m_{0}}(x, y)\right)^{2} & \leq 3\left(2 P\left[x<X_{0}^{n} \leq y\right]+|f(x, y)|^{2} E\left(Y_{0}^{0, m_{0}}\right)^{2}\right) \\
& \leq C \int_{x}^{y}\left(f^{n}(u)+\left|f^{\prime}(u)\right|\right) d u \leq C \mu_{r}(x, y)
\end{aligned}
$$

and therefore

$$
E\left(S_{N 0}^{n}(x, y)\right)^{2} \leq C N m_{0} \mu_{r}(x, y)
$$

where the constant $C$ does not depend on $N, m_{0}, n$. This proves (6.3).

To prove (6.4), we need a convenient representation of $U_{t}^{n, m}(x, y)$. Note, for any $m>n$,

$$
\begin{aligned}
P\left[X_{t}^{n} \leq x \mid \mathcal{B}_{t-m}\right] & =P\left[Y_{t}^{0, m-1}+V\left(\zeta_{t}, \ldots, \zeta_{t-n}, 0, \ldots\right)+Y_{t}^{m, \infty} \leq x \mid \zeta_{t-m}, \zeta_{t-m-1}, \ldots\right] \\
& =F^{n, m-1}\left(x-Y_{t}^{m, \infty}\right)
\end{aligned}
$$

where (recall notation) $F^{n, m}(x):=P\left[X_{t}^{n, m} \leq x\right], \quad X_{t}^{n, m}:=Y_{t}^{0, m}+V_{t}^{n}=\sum_{i=0}^{m} b_{i} \zeta_{t-i}+$ $V\left(\zeta_{t}, \ldots, \zeta_{t-n}, 0, \ldots\right)$. Then $U_{t}^{n, m}(x, y)=U_{t, 1}^{n, m}(x, y)+U_{t, 2}^{n, m}(x, y)$, where

$$
\begin{aligned}
U_{t, 1}^{n, m}(x, y):= & \int_{x}^{y} \int_{\mathbb{R}}\left\{f^{n, m-1}\left(u-b_{m} \zeta_{t-m}-Y_{t}^{m+1, \infty}\right)-f^{n, m-1}\left(u-b_{m} \tilde{z}-Y_{t}^{m+1, \infty}\right)\right. \\
\left.+b_{m}\left(\zeta_{t-m}-\tilde{z}\right)\left(f^{n, m-1}\right)^{\prime}\left(u-Y_{t}^{m+1, \infty}\right)\right\} d \Phi(\tilde{z}) d u & \\
U_{t, 2}^{n, m}(x, y):= & b_{m} \zeta_{t-m} \int_{x}^{y}\left\{f^{\prime}(u)-\left(f^{n, m-1}\right)^{\prime}\left(u-Y_{t}^{m+1, \infty}\right)\right\} d u
\end{aligned}
$$

where we used $E \zeta_{0}=\int_{\mathbb{R}} \tilde{z} d \Phi(\tilde{z})=0$. The term $U_{t, 1}^{n, m}(x, y)$ can be further rewritten as

$$
\begin{aligned}
U_{t, 1}^{n, m}(x, y)=\int_{\mathbb{R}}\left[\int_{x}^{y} d u \int_{b_{m} \tilde{z}}^{b_{m} \zeta_{t-m}} d v\right. & \left\{\left(f^{n, m-1}\right)^{\prime}\left(u-Y_{t}^{m+1, \infty}\right)\right. \\
& \left.\left.-\left(f^{n, m-1}\right)^{\prime}\left(u-v-Y_{t}^{m+1, \infty}\right)\right\}\right] d \Phi(\tilde{z}) .
\end{aligned}
$$

By Lemma 6 (5.12), the function $h(x)=\left(f^{n, m-1}\right)^{\prime}(x)$ satisfies conditions (5.6). Therefore by Lemma 5 (5.10),

$$
\begin{aligned}
& \left|\int_{x}^{y} d u \int_{b_{m} \tilde{z}}^{b_{m} \zeta_{t-m}} d v\left\{\left(f^{n, m-1}\right)^{\prime}\left(u-Y_{t}^{m+1, \infty}\right)-\left(f^{n, m-1}\right)^{\prime}\left(u-v-Y_{t}^{m+1, \infty}\right)\right\}\right| \\
& \leq C \mu_{r}(x, y)\left(\left|b_{m} \zeta_{t-m}\right|^{r}+\left|b_{m} \tilde{z}\right|^{r}\right)\left(1 \vee\left|Y_{t}^{m+1, \infty}\right|^{r}\right), \quad \text { a.s., }
\end{aligned}
$$


implying

$$
\left|U_{t, 1}^{n, m}(x, y)\right| \leq C \mu_{r}(x, y)\left|b_{m}\right|^{r}\left(1+\left|\zeta_{t-m}\right|^{r}\right)\left(1+\left|Y_{t}^{m+1, \infty}\right|^{r}\right), \quad \text { a.s.. }
$$

By independence of $\zeta_{t-m}$ and $Y_{t}^{m+1, \infty}$, and using $E\left|Y_{t}^{m, \infty}\right|^{3} \leq C<\infty$, see (6.7) below, for any $r<3 / 2$ and any $0 \leq n<m, m-n \geq j_{0}$ we obtain that

$$
E\left(U_{t, 1}^{n, m}(x, y)\right)^{2} \leq C \mu_{r}(x, y)\left|b_{m}\right|^{2 r},
$$

where $C$ is independent of $n, m$.

Next, consider

$$
\begin{aligned}
U_{t, 2}^{n, m}(x, y) & =b_{m} \zeta_{t-m} \int_{x}^{y}\left\{f^{\prime}(u)-f^{\prime}\left(u-Y_{t}^{m+1, \infty}\right)\right\} d u \\
& +b_{m} \zeta_{t-m} \int_{x}^{y}\left\{f^{\prime}\left(u-Y_{t}^{m+1, \infty}\right)-\left(f^{n, m-1}\right)^{\prime}\left(u-Y_{t}^{m+1, \infty}\right)\right\} d u \\
& =: \sum_{i=1}^{2} W_{t, i}^{n, m}(x, y) .
\end{aligned}
$$

By Lemma 6, $f^{\prime}$ satisfies (5.6), hence by Lemma 5 (5.9),

$$
\left|W_{t, 1}^{n, m}(x, y)\right| \leq C \mu_{r}(x, y)\left|b_{m} \zeta_{t-m}\right|\left(\left|Y_{t}^{m+1, \infty}\right|+\left|Y_{t}^{m+1, \infty}\right|^{r}\right), \quad \text { a.s. }
$$

By (2.3) and Rosenthal inequality, for any $1 \leq r \leq 3$ we have

$$
E\left|Y_{t}^{m+1, \infty}\right|^{2 r} \leq C\left(\beta_{m+1}^{r}+\sum_{i=m+1}^{\infty}\left|b_{i}\right|^{2 r}\right) \leq C \beta_{m+1}^{r} \leq C \beta_{m+1} .
$$

Therefore, for any $1<r<3 / 2$, we obtain

$$
E\left(W_{t, 1}^{n, m}(x, y)\right)^{2} \leq C \mu_{r}(x, y) b_{m}^{2} \beta_{m} .
$$

Finally, to estimate $W_{t, 2}^{n, m}(x, y)$, use Lemma 6 (5.13)-(5.14) together with Lemma 5 (5.7). This yields

$$
\left|W_{t, 2}^{n, m}(x, y)\right| \leq C \mu_{r}(x, y)\left|b_{m} \zeta_{t-m}\right|\left(\alpha_{n}+\beta_{m}^{1 / 2}\right)\left(1+\left|Y_{t}^{m+1, \infty}\right|^{r}\right), \quad \text { a.s. }
$$

Consequently,

$$
E\left(W_{t, 2}^{n, m}(x, y)\right)^{2} \leq C \mu_{r}(x, y) b_{m}^{2}\left(\alpha_{n}^{2}+\beta_{m}\right) .
$$

Note $\left|b_{m}\right|^{2 r}=o\left(b_{m}^{2} \beta_{m}\right)$ for $r<3 / 2$ sufficiently close to $3 / 2$. Hence and from (6.6), (6.8), (6.9) we obtain

$$
E\left(U_{t}^{n, m}(x, y)\right)^{2} \leq C \mu_{r}(x, y) b_{m}^{2}\left(\beta_{m}+\alpha_{n}^{2}\right)
$$


implying

$$
E\left(S_{N 1}^{n}(x, y)\right)^{2} \leq C \mu_{r}(x, y) \sum_{1 \leq t \leq t^{\prime} \leq N} \sum_{m=1}^{\infty}\left|b_{m}\right|\left|b_{t^{\prime}-t+m}\right|\left(\beta_{m}^{1 / 2}+\alpha_{n}\right)\left(\beta_{t^{\prime}-t+m}^{1 / 2}+\alpha_{n}\right) .
$$

Here,

$$
\begin{aligned}
\sum_{1 \leq t \leq t^{\prime} \leq N} \sum_{m=1}^{\infty}\left|b_{m}\right|\left|b_{t^{\prime}-t+m}\right| \beta_{m}^{1 / 2} \beta_{t^{\prime}-t+m}^{1 / 2} & \leq \sum_{1 \leq t \leq t^{\prime} \leq N} \sum_{m=1}^{\infty} m^{(4 d-3) / 2}\left(t^{\prime}-t+m\right)^{(4 d-3) / 2} \\
& \leq C \Theta(N),
\end{aligned}
$$

where $\Theta(N)$ is defined in (6.5). The remaining sums on the r.h.s. of (6.10) can be similarly estimated. This proves the claim (6.4) and Lemma 7, too.

\section{$7 \quad$ Proof of Lemma 2}

We shall use a chaining argument together with the following bound: for any $N \geq 1, x_{1}<x_{2}$

$$
E\left(\hat{F}_{N}\left(x_{1}, x_{2}\right)-\hat{F}_{N}^{n(N)}\left(x_{1}, x_{2}\right)\right)^{2} \leq C N^{-(\rho+11 d-13) \lambda} .
$$

Clearly, it suffices to show (7.1) for $x_{1}=-\infty, x_{2}=x$. Note $E\left(\hat{F}_{N}(x)-\hat{F}_{N}^{n}(x)\right)^{2} \leq$ $E\left(I\left(X_{0} \leq x\right)-I\left(X_{0}^{n} \leq x\right)\right)^{2}=: q(x)$ by Cauchy-Schwarz inequality. Put $X:=X_{0}, X^{n}:=$ $X_{0}^{n}$. By Minkowski inequality,

$$
q(x)=E\left(\sum_{k=n+1}^{\infty}\left(I\left(X^{k} \leq x\right)-I\left(X^{k-1} \leq x\right)\right)\right)^{2} \leq\left(\sum_{k=n+1}^{\infty} q_{k}^{1 / 2}(x)\right)^{2},
$$

where

$$
q_{k}(x):=E\left(I\left(X^{k} \leq x\right)-I\left(X^{k-1} \leq x\right)\right)^{2} .
$$

Recall $X^{k}=Y^{0, k}+V^{k}+Y^{k+1, \infty}$, where $Y^{k+1, \infty}=\sum_{i=k+1}^{\infty} b_{i} \zeta_{-i}$ is independent of $Y^{0, k}+V^{k}$. Then

$$
q_{k}(x)=E \int_{\mathbb{R}}\left(I_{\left(-\infty, x-V^{k}\right]}(y)-I_{\left(-\infty, x-V^{k-1}\right]}(y)\right)^{2} g^{k+1, \infty}\left(y-Y^{0, k}\right) d y .
$$

Note for any $x, a<b$

$$
\int_{\mathbb{R}}\left(I_{(-\infty, x-a]}(y)-I_{(-\infty, x-b]}(y)\right)^{2} d y=|a-b| .
$$

Therefore by $\sup _{x} g^{k+1, \infty}(x) \leq C k^{\theta}$, see Lemma 4 , we obtain

$$
\left|q_{k}\left(x_{1}, x_{2}\right)\right| \leq C k^{\theta} E\left|V^{k}-V^{k-1}\right| \leq C k^{\theta} \gamma_{k} \leq C k^{\theta-\rho} .
$$


Thus, $q\left(x_{1}, x_{2}\right) \leq C\left(n^{1+(\theta-\rho) / 2}\right)^{2}=C n^{2+\theta-\rho}$, where $\theta=11(1-d)$. This proves (7.1).

Next we describe chaining. For any integer $k \geq 1$ define the partition

$$
-\infty=: \pi_{0, k}<\pi_{1, k}<\cdots<\pi_{2^{k}-1, k}<\pi_{2^{k}, k}=:+\infty
$$

such that

$$
\mu_{r}\left(\pi_{j, k}, \pi_{j+1, k}\right)=\mu_{r}(\mathbb{R}) 2^{-k}, \quad j=0,1, \ldots, 2^{k}-1 .
$$

Here, $\mu_{r}(x, y)=\int_{x}^{y}(1+|u|)^{-r} d u$ is the same measure as in the formulation of Lemma 7 ; $\mu_{r}(\mathbb{R})=\int_{\mathbb{R}}(1+|u|)^{-r} d u$. Let $K=K(N)=O(\log N)$ be an integer which will be specified below. For any $x \in \mathbb{R}$ and any $k=0,1, \ldots, K$, define $j_{k}^{x}$ by

$$
\pi_{j_{k}^{x}, k} \leq x<\pi_{j_{k}^{x}+1, k}
$$

Put $V_{N}(x):=N^{(1 / 2)-d}\left(\hat{F}_{N}(x)-\hat{F}_{N}^{n(N)}(x)\right)$. Then

$$
V_{N}(x)=V_{N}\left(\pi_{j_{K}^{x}, K}\right)+V_{N}\left(\pi_{j_{K}^{x}, K}, x\right) .
$$

By definition of $V_{N}$, for any $y<w<z$,

$$
\begin{aligned}
V_{N}(y, w) & \leq N^{(1 / 2)-d} \hat{F}_{N}(y, z)-N^{(1 / 2)-d} \hat{F}_{N}^{n(N)}(y, w) \\
& =N^{(1 / 2)-d}\left(\hat{F}_{N}(y, z)-\hat{F}_{N}^{n(N)}(y, z)\right)+N^{(1 / 2)-d} \hat{F}_{N}^{n(N)}(w, z) \\
& \leq\left|V_{N}(y, z)\right|+2 \sup _{x \in \mathbb{R}} N^{(1 / 2)-d}\left|\hat{F}_{N}^{n(N)}(x)-F^{n(N)}(x)+f(x) \bar{Y}_{N}\right| \\
& +\sup _{y<w<z} N^{(1 / 2)-d}\left(F^{n(N)}(w, z)+f(w, z)\left|\bar{Y}_{N}\right|\right) .
\end{aligned}
$$

In a similar way,

$$
\begin{aligned}
V_{N}(y, w) & \geq-N^{(1 / 2)-d} \hat{F}_{N}^{n(N)}(y, z) \\
& \geq-2 \sup _{x \in \mathbb{R}} N^{(1 / 2)-d}\left|\hat{F}_{N}^{n(N)}(x)-F^{n(N)}(x)+f(x) \bar{Y}_{N}\right| \\
& -\sup _{y<w<z} N^{(1 / 2)-d}\left(F^{n(N)}(w, z)+f(w, z)\left|\bar{Y}_{N}\right|\right) .
\end{aligned}
$$

The above bounds combine to

$$
\sup _{y<w<z}\left|V_{N}(y, w)\right| \leq\left|V_{N}(y, z)\right|+2 R_{N}+W_{N}(y, z),
$$

where

$$
\begin{aligned}
R_{N} & :=\sup _{x \in \mathbb{R}} N^{(1 / 2)-d}\left|\hat{F}_{N}^{n(N)}(x)-F^{n(N)}(x)+f(x) \bar{Y}_{N}\right|, \\
W_{N}(y, z) & :=N^{(1 / 2)-d}\left(F^{n(N)}(y, z)+\left|\bar{Y}_{N}\right| \int_{y}^{z}\left|f^{\prime}(u)\right| d u\right) .
\end{aligned}
$$


Thus,

$$
\sup _{x}\left|V_{N}\left(\pi_{j_{K}^{x}, K}, x\right)\right| \leq \max _{1 \leq j \leq 2^{K}}\left|V_{N}\left(\pi_{j-1, K}, \pi_{j, K}\right)\right|+2 R_{N}+\max _{1 \leq j \leq 2^{K}} W_{N}\left(\pi_{j-1, K}, \pi_{j, K}\right) .
$$

Then from (7.2) we obtain

$$
\begin{aligned}
\sup _{x}\left|V_{N}(x)\right| & \leq 2 R_{N}+\max _{0 \leq j \leq 2^{K}-1}\left|V_{N}\left(\pi_{j, K}\right)\right|+\max _{1 \leq j \leq 2^{K}}\left|V_{N}\left(\pi_{j-1, K}, \pi_{j, K}\right)\right| \\
& +\max _{1 \leq j \leq 2^{K}} W_{N}\left(\pi_{j-1, K}, \pi_{j, K}\right) .
\end{aligned}
$$

Here, $R_{N}=o_{p}(1)$ by Lemma 7 . Next, by (7.1),

$$
\begin{aligned}
P\left(\max _{0 \leq j \leq 2^{K}-1}\left|V_{N}\left(\pi_{j, K}\right)\right|>\delta\right) & \leq \sum_{j=0}^{2^{K}-1} P\left(\left|V_{N}\left(\pi_{j, K}\right)\right|>\delta\right) \\
& \leq \delta^{-2} \sum_{j=0}^{2^{K}-1} E\left|V_{N}\left(\pi_{j, K}\right)\right|^{2} \\
& \leq C \delta^{-2}\left(2^{K}+1\right) N^{1-2 d-(\rho+11 d-13) \lambda} .
\end{aligned}
$$

Choose $K=\left[\log _{2} N^{(1 / 2)-d+\epsilon}\right]$, where $\epsilon>0$ is small enough. Then the r.h.s. of (7.4) tends to 0 in view of the inequality on $\lambda$ in the formulation of Lemma 2. Therefore the second term on the r.h.s. of (7.3) is $o_{p}(1)$. The third term is treated exactly the same way.

Consider the last term on the r.h.s. of (7.3). By Lemma $6, W_{N}(x, y) \leq C \mu_{r}(x, y) N^{(1 / 2)-d}(1+$ $\left.\left|\bar{Y}_{N}\right|\right)$, implying

$$
E \max _{1 \leq j \leq 2^{K}} W_{N}\left(\pi_{j-1, K}, \pi_{j, K}\right) \leq C 2^{-K} N^{(1 / 2)-d} E\left(1+\left|\bar{Y}_{N}\right|\right) \leq C 2^{-K} N^{(1 / 2)-d}=o(1),
$$

with the above choice of $K$. This proves $\sup _{x \in \mathbb{R}}\left|V_{N}(x)\right|=o_{p}(1)$.

Finally,

$$
\begin{aligned}
\left|F(x)-F^{n}(x)\right| & =\left|E\left(I\left(X_{0} \leq x\right)-I\left(X_{0}^{n} \leq x\right)\right)\right| \\
& \leq E^{1 / 2}\left(I\left(X_{0} \leq x\right)-I\left(X_{0}^{n} \leq x\right)\right)^{2} \\
& =(q(x))^{1 / 2} \leq C n^{1+(\theta-\rho) / 2}=C n^{-(\rho+11 d-13) / 2}
\end{aligned}
$$

as above, uniformly in $x \in \mathbb{R}$. Clearly, if $\lambda$ is chosen as in Lemma 2, this implies $N^{(1 / 2)-d} \sup _{x \in \mathbb{R}}\left|F^{n(N)}(x)-F(x)\right|=o(1)$. This completes the proof of Lemma 2. 


\section{References}

Davydov, Yu.A. (1970) The invariance principle for stationary processes. Theory Probab. Appl. 15, 487-498.

Dehling, H., Taqqu, M. S. (1989) The empirical process of some long-range dependent sequences with an application to $U$-statistics. Ann. Stat. 17, 1767-1783.

Diaconis, P., Freedman, D. (1999) Iterated random functions. SIAM Review 41, 45-76.

Dobrushin, R.L., Major, P. (1979) Non-central limit theorems for non-linear functions of Gaussian fields. Z. Wahrsch. verw. Geb. 50, 27-52.

Doukhan, P. (1994) Mixing: Properties and Examples. Lecture Notes in Statistics vol. 85, Springer-Verlag, New York.

Doukhan, P. (2002) Models, inequalities and limit theorems for stationary sequences. In: Taqqu, M.S., Oppenheim, G., Doukhan, P. (eds.) Long-Range Dependence: Theory and Applications, pp. 43-100. Birkhaüser, Boston.

Doukhan, P., Lang, G., Surgailis, D. (2002) Asymptotics of weighted empirical processes of linear fields with long-range dependence. Ann. Inst. Henri Poincaré Probabilités et Statistiques 38, 879-896.

Duflo, M. (1990) Méthodes récursives aléatoires. Masson, Paris. (English Edition: Springer-Verlag, 1996)

Giraitis, L., Kokoszka, P., Leipus, R. (2000) Stationary ARCH models: dependence structure and CLT. Econometric Theory 16, 3-22.

Giraitis, L., Koul, H.L., Surgailis, D. (1996) Asymptotic normality of regression estimators with long memory errors. Statist. Probab. Letters 29, 317-335.

Giraitis, L., Surgailis, D. (1999) Central limit theorem for the empirical process of a linear sequence with long memory. J. Stat. Plan. Inf. 80, 81-93.

Giraitis, L., Surgailis, D. (2002) ARCH-type bilinear models with double long memory. Stoch. Process. Appl. 100, 275-300.

Ho, H.-C., Hsing, T. (1996) On the asymptotic expansion of the empirical process of long memory moving averages. Ann. Statist. 24, 992-1024. 
Kallenberg, O. (1997) Foundations of Modern Probability. Springer-Verlag, New-York.

Robinson, P.M. (1991) Testing for strong serial correlation and dynamic conditional heteroskedasticity in multiple regression. J. Econometrics 47, 67-84.

Rosenblatt, M. (1961) Independence and dependence. In: Proc. Fourth Berkeley Symp. Math. Statist. Probab., pp. 411-443. Univ. California Press, Berkeley.

Surgailis, D. (2000) Long-range dependence and Appell rank. Ann. Probab. 28, 478-497.

Taqqu, M.S. (1975) Weak convergence to fractional Brownian motion and to the Rosenblatt process. Z. Wahrsch. verw. Geb. 31, 287-302. 